Check for updates

Cite this: RSC Adv., 2017, 7, 37302

\title{
A push-pull silafluorene fluorophore for highly efficient luminescent solar concentrators $\uparrow$
}

\author{
Federico Gianfaldoni, ${ }^{a}$ Francesca De Nisi, (D) a Giuseppe lasilli, ${ }^{a}$ Annamaria Panniello, \\ Elisabetta Fanizza, ${ }^{\text {bc }}$ Marinella Striccoli, ${ }^{b}$ Daiki Ryuse, ${ }^{d}$ Masaki Shimizu, (D) d \\ Tarita Biver (iD ${ }^{a}$ and Andrea Pucci (iD *a
}

\begin{abstract}
We report on the preparation of luminescent collectors based on poly(methyl methacrylate) (PMMA) thin films doped with a red-emitting 2-amino-7-acceptor-9-silafluorene, where the amino group is $-\mathrm{N}\left(\mathrm{CH}_{3}\right)_{2}$ and the acceptor is $-\mathrm{CH}=\mathrm{C}(\mathrm{CN})_{2}$. The results obtained from photophysical investigations of the dye in different solvents and in PMMA are very encouraging as the silafluorene dyes turn out to be highly dispersed in the solid matrix, stable upon irradiation and highly emissive. QY and lifetime investigations demonstrate that autoabsorption phenomena moderately occur with SilaFluo content, and the optical features still maintained very significant, also at the highest fluorophore concentration $(Q Y \sim$ $65 \%$ ). Study of the LSCs features yields excellent optical efficiencies of $9.6 \%$ attained for $25 \mu \mathrm{m}$ thick PMMA films containing the $1.5 \mathrm{wt} \%$ of SilaFluo. This performance is at the top level with respect to the current state-of-art of similar devices based on perylene-based fluorophores such as Lumogen Red.
\end{abstract}

Received 20th July 2017

DOI: $10.1039 / \mathrm{c} 7 \mathrm{ra0} 0822 \mathrm{k}$

rsc.li/rsc-advances

incorporated in a dielectric plastic matrix transparent to most of solar radiation. Notably, LSCs are slabs of large surface exposed to the incident radiation, compared to the smaller side surface at which a fraction of the emitted photons is trapped due to the total internal reflection. ${ }^{4}$ Photons collected at the edges of the LSC are transmitted to a solar PV cell attached at the edge of the LSC collector to produce electric power. Red-emitting fluorophores are typically preferred since their fluorescence well matches the Si-based PV cells band gap. ${ }^{8-10}$ Nevertheless, these fluorophores are generally characterized by relatively low QY values in the solid state, thus adversely affecting LSCs performances. ${ }^{11}$ The maximum power conversion efficiency for a LSC-PV system was recorded for poly(methyl methacrylate) (PMMA) embedding perylenebased fluorophores. ${ }^{10,12,13}$ Lumogen F Red 305 is considered the state-of-the-art for LSCs ${ }^{4,14}$ due to its very high QY (about 97\% in PMMA) $)^{15}$ and outstanding photostability. Nevertheless, its very small Stokes shift combined with its recent quotation at $7500 €$ per kg might definitely affect LSCs technology diffusion in the BIPV market. As recently reviewed by McKenna and Evans, ${ }^{14}$ different strategies aimed at maximising LSCs performances have been already accomplished, most of them focused on the use of different kind of redemitting fluorophores characterized by large Stokes shift and negligible aggregation induced quenching. ${ }^{16-22}$ In the continuous search for novel alternatives to perylene-based fluorophores, $\pi$-conjugated silafluorenes have attracted considerable attention owing to their easy synthesis, potential applications in organic electronics and efficient emission in a wide range of the visible spectrum..$^{23-30}$ Notably, 2-donor-7-

\footnotetext{
${ }^{a}$ Department of Chemistry and Industrial Chemistry, University of Pisa, via Moruzzi 13, 56124 Pisa, Italy.E-mail: andrea.pucci@unipi.it

${ }^{b}$ CNR-IPCF Bari Unit, c/o Department of Chemistry, via Orabona 4, 70126 Bari, Italy 'Department of Chemistry, University of Bari, via Orabona 4, 70126 Bari, Italy ${ }^{d}$ Faculty of Molecular Chemistry and Engineering, Kyoto Institute of Technology, 1 Hashikami-cho, Matsugasaki, Sakyo-ku, Kyoto 606-8585, Japan

$\dagger$ Electronic supplementary information (ESI) available. See DOI: $10.1039 / \mathrm{c} 7 \mathrm{ra} 08022 \mathrm{k}$
} 
acceptor-9-silafluorenes have been found to exhibit remarkable fluorescence properties and highly efficient emission also in the solid state (e.g., a neat thin film and a doped polymer film). ${ }^{31}$

In this regard, we have selected a recently developed redemitting $\mathrm{Me}_{2} \mathrm{~N} / \mathrm{CH}=(\mathrm{CN})_{2}$-substituted 9-silafluorene, abbreviated as SilaFluo (Fig. 1), based on the silafluorene core and efficiently decorated with donor (push) dimethylamino and acceptor (pull) 2,2-dicyanoethenyl moieties. SilaFluo displayed red fluorescence $\left(\lambda_{\mathrm{em} \text { max }}=605 \mathrm{~nm}\right.$ ) with an excellent quantum yield (up to 71\%) when dispersed in PMMA films, thus shedding light on the high potential of this fluorophore to be used for the preparation of high efficiency LSCs.

\section{Experimental}

\section{Materials}

All the solvents were purchased from Sigma-Aldrich and used as received. Poly(methyl methacrylate) (PMMA, Aldrich, $M_{\mathrm{w}}=350000 \mathrm{~g} \mathrm{~mol}^{-1}$, acid number $<1 \mathrm{mg} \mathrm{KOH} \mathrm{per} \mathrm{g}$ ) and Lumogen Red F350 (LR, BASF) were used as received. SilaFluo was prepared following the synthetic procedures reported in the literature. ${ }^{31}$ Optically clear glass slides were prepared by cleaning in $6 \mathrm{M} \mathrm{HCl}$ for $12 \mathrm{~h}$, rinsing with water, acetone and 2-propanol and then drying for $8 \mathrm{~h}$ at $120{ }^{\circ} \mathrm{C}$.

\section{Preparation of SilaFluo/PMMA and LR/PMMA films}

Different fluorophore/PMMA thin films were prepared by pouring about $0.8 \mathrm{~mL}$ chloroform $\left(\mathrm{CHCl}_{3}\right)$ solution containing about $30 \mathrm{mg}$ of PMMA and the desired amount of fluorophore (i.e., SilaFluo or LR) to get concentrations in the range of 0.1-1.5 wt $\%$ on $35 \times 50 \mathrm{~mm}$ glass surface. $\mathrm{CHCl}_{3}$ evaporation was obtained on a warm plate $\left(30^{\circ} \mathrm{C}\right)$ and in a closed environment. The film thickness was measured to be $25 \pm 5 \mu \mathrm{m}$ (Starrett micrometer). The polymer films were then removed after immersion in water and stored in a desiccator for successive measurements by attaching them on $50 \times 50 \times 3 \mathrm{~mm}$ cleaned glass (Edmund Optics Ltd BOROFLOAT window $50 \times 50 \mathrm{TS}$ ) with a high-purity silicone oil (poly(methylphenyl siloxane), 710 fluid, Aldrich, $n=1.5365$ ). Negligible differences of the optical features were recorded compared with the freshly prepared ones.

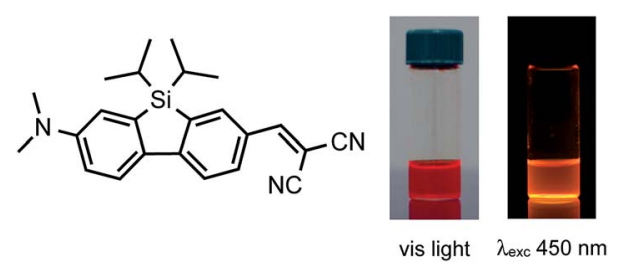

Fig. 1 Chemical structure of SilaFluo (left) and photos (right) of its $10^{-3} \mathrm{M} \mathrm{CHCl}_{3}$ solution under visible light and at $450 \mathrm{~nm}$ by means of a Dark Reader 46B transilluminator.

\section{Characterization}

Spectrophotometric measurements were performed using a Shimadzu UV2450 double beam spectrophotometer and a Perkin-Elmer Lambda 650 spectrometer with temperature control to within $\pm 0.1{ }^{\circ} \mathrm{C}$. The fluorescence measurements in solution were performed using a Perkin Elmer LS55 spectrofluorometer with temperature control to within $\pm 0.1^{\circ} \mathrm{C}$. Relative quantum yield (QY) values in solution were calculated according to a procedure that uses a standard as a reference (the SilaFluo dye itself in diethyl ether, $\left.\mathrm{QY}_{\mathrm{ST}}=0.56\right){ }^{31}$ the equation used is $\mathrm{QY}_{\mathrm{X}}=\mathrm{QY}_{\mathrm{ST}}\left(\operatorname{Grad}_{\mathrm{X}} / \operatorname{Grad}_{\mathrm{ST}}\right)\left(\eta_{\mathrm{X}} / \eta_{\mathrm{ST}}\right)^{2}$ where $\operatorname{Grad}_{i}$ is the slope of a fluorescence area $\left(\lambda_{\text {exc }} 450 \mathrm{~nm}\right.$, slides $\left.2.5 \mathrm{~nm}\right) v s$. absorbance $(\lambda=450 \mathrm{~nm})$ plot for the $i$-th species (ST $=$ standard, $\mathrm{X}=$ dye), whereas $\eta$ is the refraction index of the solvent $\left(\eta_{\mathrm{ST}}=1.352\right)$.

Fluorescence spectra in the solid state were measured at room temperature by a Horiba Jobin-Yvon Fluorolog®-3 spectrofluorometer equipped with a $450 \mathrm{~W}$ Xenon arc lamp and double-grating both excitation and emission monochromators. The emission quantum yields of the solid samples were obtained by means of a $152 \mathrm{~mm}$ diameter "Quanta-phi" integrating sphere, coated with Spectralon ${ }^{\circledR}$ and mounted in the optical path of the spectrofluorometer, using as excitation source the $450 \mathrm{~W}$ Xenon lamp coupled with a double-grating monochromator for selecting wavelengths. Time-resolved PL measurements were performed by using the time-correlated single photon counting (TCSPC) technique, with an HORIBA Jobin-Yvon FluoroHub. The experimental setup was equipped with a laser diode (NanoLED $485 \mathrm{~L}$ ) emitting $\approx 200$ ps pulses at $485 \mathrm{~nm}$ with a repetition rate of $1 \mathrm{MHz}$. The PL was dispersed by a double grating monochromator and detected by a picosecond photon counter (TBX ps Photon Detection Module, HORIBA Jobin-Yvon) with a temporal resolution of about 200 ps. Micrographs of films at diverse SilaFluo loading were recorded by bright field and fluorescence microscopy with a Carl Zeiss AXIO direct microscope equipped with an AxioCam MRc 5 . Fluorescence acquisitions were performed using a filter set with an excitation range between 450 and $490 \mathrm{~nm}$ and a transmission pass band $>515 \mathrm{~nm}$. Field emission scanning electron microscopy (FE-SEM) was performed by a Zeiss Sigma microscope operating in the range $0.5-20 \mathrm{kV}$ and equipped with an in-lens secondary electron detector. Samples were mounted onto stainless-steel sample holders by a double-sided carbon tape and covered by a thin layer $(5 \mathrm{~nm})$ of Cr by a sputtering process under $\mathrm{Ar}^{+}$.

\section{Optical efficiency measurement}

A home-built equipment analogous to earlier setups ${ }^{\mathbf{9 , 2 0 , 3 2}}$ was utilized to measure the efficiency of the LSCs. For each fluorophore concentration, three LSCs were prepared and tested. The system is composed by a wooden box with darkened walls and a white back scattering layer (ERGA TAPES Srl Microcellular MCPET reflective sheet). The sample holder with the photovoltaic (PV) module (IXYS SLMD121H08L mono solar cell $86 \times$ $14 \mathrm{~mm}: V_{\mathrm{oc}}=5.04 \mathrm{~V}, I_{\mathrm{sc}}=50.0 \mathrm{~mA}, \mathrm{FF}>70 \%, \eta=22 \%$, Fig. S1 $\dagger$ ) is placed $2.5 \mathrm{~cm}$ above the scattering layer. The PV cell is 
masked with black tape to match the LSC edge $(50 \times 3 \mathrm{~mm})$ so that limiting the stray light to negligible levels. The other three edges of the LSC were covered with a reflective aluminum tape. A led lamp (12 V DC $5 \mathrm{~W}$, colour $T=5500 \mathrm{~K}$ ) was housed $10 \mathrm{~cm}$ above the sample and with the main body located outside the box to prevent its heating-up. The PV module is connected to a digital potentiometer (AD5242) controlled via $\mathrm{I}^{2} \mathrm{C}$ by an Arduino Uno (https://www.arduino.cc) microcontroller using $\mathrm{I}^{2} \mathrm{C}$ master library. A digital multimeter (KEITHLEY 2010) is connected in series with the circuit, between the PV module and the potentiometer, to collect the current as a function of the external load (Fig. $\mathrm{S} 2 \dagger$ ). Conversely, the voltage is measured by connecting the multimeter in parallel to the digital potentiometer (Fig. S3†). Arduino Uno controls the multimeter via SCPI language over RS-232 bus using a TTL to RS-232 converter chip (MAX232). Arduino Uno is connected to pe via USB port and controlled by a Python script. A 12 V DC voltage light source was selected so that the luminous flux is continuous and there is no need to compensate the PV module capacitance. The measurement cycle begins with a signal from PC to Arduino which sets the multimeter parameter to measure current. Then Arduino begins the measure loop: (1) set the potentiometer to a given value; (2) send trigger signal to the multimeter; (3) read the measured data; and (4) send the data back to PC. The loop is repeated 256 times for potentiometer values ranging $60 \Omega$ to 1 $\mathrm{M} \Omega$. Arduino sets the multimeter to measure voltage and for each potentiometer value the system records 8 data samples which are subsequently processed by the Python script. The optical efficiency is reported as $\eta_{\text {opt }}$ and obtained from the concentration factor, which is the ratio between the maximal current of the PV cell attached the LSC edges under illumination of a light source and the maximal current of the bare cell put perpendicular to the light source. In our setup, the QE variation of the $\mathrm{PV}$ module is under $2 \%$ within the emission range of SilaFluo from $500 \mathrm{~nm}$ to $700 \mathrm{~nm}$. Measurements were repeated on freshly prepared new LSCs if QE variations were higher than $2 \%$.

\section{Results and discussion}

\section{Spectroscopic characterisation of SilaFluo in solution}

SilaFluo has been recently designed, synthesized and characterised as red-emitting push-pull 9-silafluorene derivative. ${ }^{31}$ Therefore, we explored SilaFluo as an effective fluorophore for the fabrication of high efficiency LSCs. In this study, the spectroscopic properties of SilaFluo were first surveyed in solvents with refractive index $(\eta)$ close to that of $\operatorname{PMMA}(\eta=1.5)$. To this aim, toluene $(\eta=$ 1.496), chloroform $(\eta=1.450)$, dioxane $(\eta=1.418)$ and diethyl ether $(\eta=1.352)$ were taken into account. Both light absorbance and emission of SilaFluo were measured in these solvents. The results obtained are collected in Table 1, whereas absorbance and fluorescence emission spectra are provided as Fig. S4 (ESI $\dagger$ ). The data confirm the strong solvatochromism of SilaFluo, as some shift of the absorbance and fluorescence emission bands still occurs, even in these quite similar solvents. The Stokes shifts (SS) are large, suggesting low auto-absorption. Absorbance spectra were recorded for different SilaFluo concentrations, so that extinction coefficients $(\varepsilon)$ could be evaluated (Table 1) for quantum yield calculations. The high $\varepsilon$ values are related to high light absorption properties. Same experiments enabled to enlighten the excellent linearity of absorbance/concentration plots (Fig. S5 $\dagger$ ). These results, together with the constancy of the ratio between the absorbance at two different wavelengths (Fig. S6†) definitely discard the possibility of some dye aggregation. Note that spectra recorded at high dye concentration ([dye] $=1.4 \times 10^{-3} \mathrm{M}, 1 \mathrm{~mm}$ light path, not shown) are very consistent with more diluted ones. No self-aggregation tendency helps homogeneous dispersion in PMMA. Fluorescence spectra at different SilaFluo concentration (ca. $2.3 \times 10^{-7}$ to $2.3 \times 10^{-6} \mathrm{M}$ range) are also done, with excitation equal to $450 \mathrm{~nm}$. Under these circumstances, emission band area/absorbance plots (Fig. S7†) enable to calculate the gradient to be used for quantum yield (QY) evaluation (Table 1), according to the procedure described in the Experimental part. Furthermore, the stability of the fluorophore was checked by measuring in time ( 3 hours) the emission upon excitation at the maximum absorbance, according to the procedure reported in literature. ${ }^{33}$ It turns out that SilaFluo is reasonably stable under the considered conditions, the measured fluorescence being unchanged for diethyl ether and chloroform, while diminished of ca. $10 \%$ in dioxane and chloroform (Fig. S8 $\dagger$ ).

Overall, the characterisation in solvents with properties similar to PMMA confirms that the SilaFluo dye constitutes a very good candidate for LSC applications with solvatochromic properties, high absorption coefficients, high Stokes shift, high quantum yield and good stability of light emission upon irradiation.

\section{Spectroscopic characterization of SilaFluo/PMMA films}

SilaFluo was dispersed at different contents (0.1-1.5 wt\%) in poly(methyl methacrylate) (PMMA), that is a commercially

Table 1 Spectroscopic characterisation of SilaFluo in solvents with refractive index $(\eta)$ close to that of PMMA $(\eta=1.5) ; T=25.0{ }^{\circ} \mathrm{C}^{a}$

\begin{tabular}{|c|c|c|c|c|c|c|c|c|}
\hline Solvent & $\eta$ & $\varepsilon$ & $\lambda_{\max }^{\mathrm{Abs}}(\mathrm{nm})$ & $\lambda_{\max }^{\text {Fluo }}(\mathrm{nm})$ & $\mathrm{SS}(\mathrm{nm})$ & $10^{-4} \times \varepsilon^{\max }\left(\mathrm{M}^{-1} \mathrm{~cm}^{-1}\right)$ & $10^{-4} \times \varepsilon^{450}\left(\mathrm{M}^{-1} \mathrm{~cm}^{-1}\right)$ & QY \\
\hline Toluene & 1.496 & 2.38 & 478 & 551 & 73 & $3.27 \pm 0.01$ & $2.58 \pm 0.01$ & 0.56 \\
\hline Chloroform & 1.450 & 4.81 & 492 & 591 & 99 & $3.67 \pm 0.02$ & $2.31 \pm 0.01$ & 0.33 \\
\hline Diethyl ether & 1.352 & 4.34 & 469 & 565 & 96 & $3.91 \pm 0.02$ & $3.51 \pm 0.02$ & 0.56 \\
\hline
\end{tabular}

${ }^{a} \eta=$ refractive index, $\varepsilon=$ dielectric constant, $\lambda_{\max }^{\mathrm{Abs}}=$ maximum of light absorbance, $\lambda_{\max }^{\text {Fluo }}=$ maximum of light emission at excitation equal to $\lambda_{\max }^{\mathrm{Abs}}, \mathrm{SS}=$ Stokes Shift $=\lambda_{\max }^{\text {fluo }}-\lambda_{\max }^{\mathrm{Abs}}, \varepsilon^{\max }=$ extinction coefficient at $\lambda_{\mathrm{ex}}=\lambda_{\max }^{\mathrm{Abs}} ; \varepsilon^{450}=$ extinction coefficient at $450 \mathrm{~nm}$. QY $=\mathrm{quantum}$ yield. 
available, transparent and amorphous polymer, i.e. possesses fundamental characteristics for LSC applications. These concentrations allowed to obtain homogeneous thin polymer films of a thickness of $25 \pm 5 \mu \mathrm{m}$ with negligible phaseseparation at the film surface even at the highest SilaFluo content, as demonstrated both by the SEM micrographs in Fig. S9† and by the fluorescence images of the films (Fig. 2). Only at the highest loading (1.6 wt\%) a faint aggregation of the fluorophore is observed. Therefore, no higher SilaFluo content in PMMA was investigated since phase separation adversely affect fluorescence collection. ${ }^{21}$

In Fig. 3a, the absorption characteristics of SilaFluo/PMMA films are reported. SilaFluo in PMMA shows a molecular absorption maxima at about $480 \mathrm{~nm}$ with a broad absorption band from 400-550 nm, which results beneficial in harvesting photons in the visible range for the LSC final application. Notably, the spectra appear very similar to that collected in solution and no evident absorption bands attributed to the formation of SilaFluo supramolecular aggregates are observed,
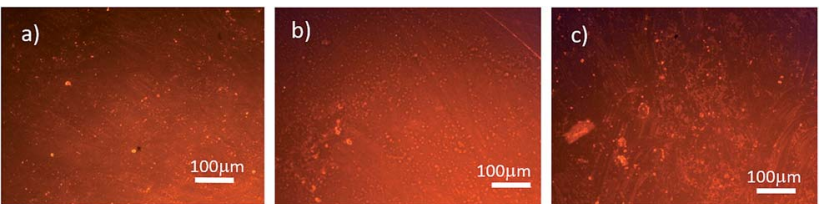

Fig. 2 Fluorescence images of PMMA (a) and PMMA/Silafluo films at different dye loading: $0.2 \%$ (a), $1.0 \%$ (b) and $1.6 \%$ (c) wt $\%$.

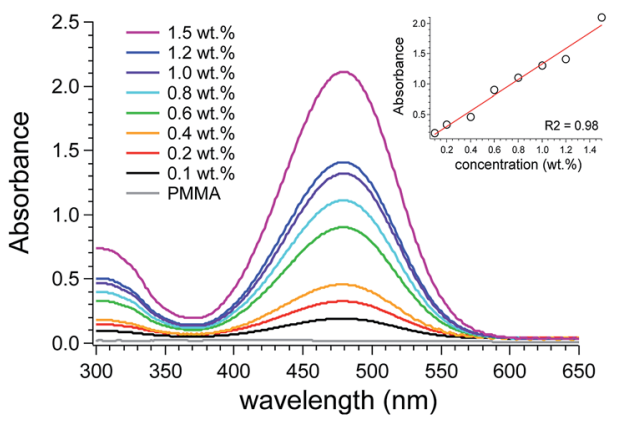

(a)

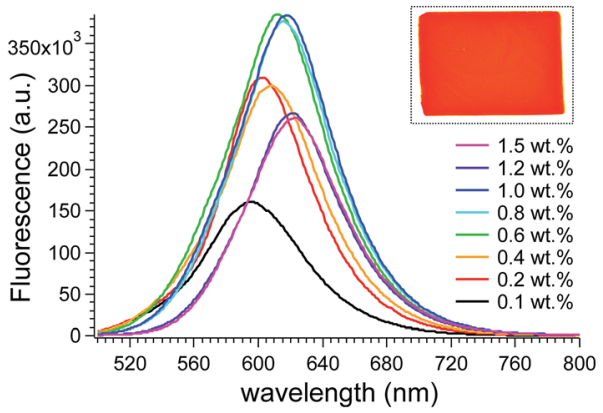

(b)

Fig. 3 (a) UV-vis absorption spectra of SilaFluo/PMMA films as a function of fluorophore concentration (wt\%). In the inset, the absorbance maximum at $480 \mathrm{~nm}$ was plotted as a function of the SilaFluo content. (b) Fluorescence spectra of the same SilaFluo/PMMA films with excitation wavelength of $480 \mathrm{~nm}$. In the inset was reported a picture of the 1.5 wt\% SilaFluo/PMMA film excited with a Dark Reader 46B transilluminator ( $\sim 450 \mathrm{~nm})$. thus suggesting a high degree of compatibility between the fluorophore and the polymer matrix. The isopropyl groups of SilaFluo are actually oriented perpendicular to the silafluorene skeleton, thus hindering the close packing of chromophores via $\pi-\pi$ stacking. ${ }^{31}$ Indeed, absorbance intensities increase regularly and with a linear trend with SilaFluo content (Fig. 3a, inset), and without leveling off at the highest concentration.

Conversely, SilaFluo/PMMA films display emission features affected by fluorophore content (Fig. 3b). When dispersed at low concentration in PMMA (i.e., $0.1 \mathrm{wt} \%$ ), SilaFluo shows fluorescence peaked at $596 \mathrm{~nm}$ with a large Stokes shift of $116 \mathrm{~nm}$. Notably, this emission range appears optimal for LSCs providing maximum spectral overlap with the external quantum efficiency of the Si-based PV cell (Fig. S1 $\dagger$ ). The fluorescence intensity increased with SilaFluo content up to $0.6-0.8 \mathrm{wt} \%$, above which fluorescence maintained its maximum intensity up to $1.0 \mathrm{wt} \%$ of fluorophore. Above this content, fluorescence quenching occurred although to a certain extent. The cause of the partial fluorescence quenching was addressed to autoabsorption phenomena, as also proved by the progressive red-shift of the emission maxima with SilaFluo content. ${ }^{22,34}$ Nevertheless, the red emission of the SilaFluo/PMMA film containing the maximum fluorophore concentration (i.e., $1.5 \mathrm{wt} \%$ ) still appeared very vivid (Fig. 3b, inset).

The lifetimes of SilaFluo/PMMA films at different fluorophore content were measured at room temperature (Fig. 4).

In Fig. 4 the recombination dynamics of the films, at increasing concentration of the fluorophore, are reported. As clearly observed in the (b) panel, it is possible to best fit the

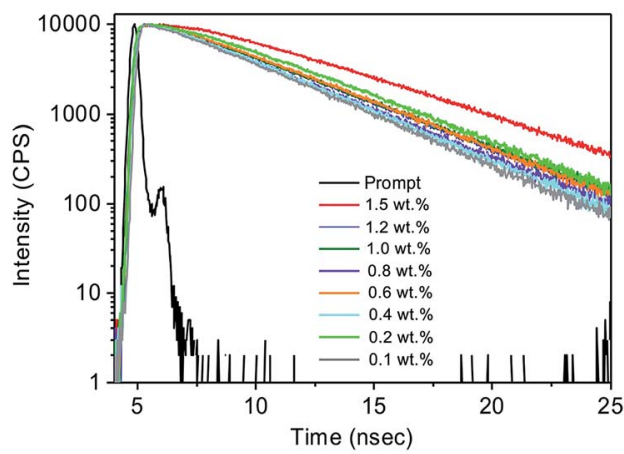

(a)

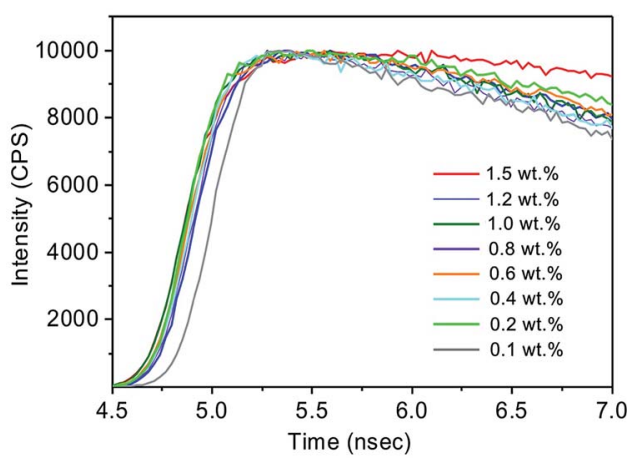

(b)

Fig. 4 (a) Temporal decay profiles of the PL of SilaFluo/PMMA films as a function of concentration at the max emission wavelength (under excitation at $485 \mathrm{~nm}$ ); (b) the initial $7 \mathrm{~ns}$ of PL buildup and decay with the PL intensity shown on a linear scale. 
transient PL decays as the sum of exponential decays, featuring an initial rise time component $\left(\tau_{\text {rise }}\right)$ that is expressed with a negative pre-exponential factor $(A 1<0)$ and a single long-lived $\tau_{\text {decay }}$ component for all films as:

$$
I(t)=A 1 \exp \left(-t / \tau_{\text {rise }}\right)+A 2 \exp \left(-t / \tau_{\text {decay }}\right)
$$

The fitting parameters are reported in Table S1. $\dagger$

It is worth to note that the negative pre-exponential factor results in a rise in intensity, which can be recognized as a distinctive sign of an excited-state reaction. ${ }^{35}$ Indeed, in the time domain, the characteristics of an excited-state reaction are a rise time in the time dependent intensities, and a negative preexponential factor in the multi-exponential analysis. Here, such a behaviour can be attributed to the donor-acceptor nature of the SilaFluo, resulting in a intramolecular excited state process. Indeed, the acceptor part of the fluorophore can be excited by two routes: by direct excitation and by energy transfer from the donor.

The rise time reflects the energy transfer time and it is manifested in the transient decays as an initial buildup in PL intensity followed by a brief plateau. As reported in Table $\mathrm{S} 1, \dagger$ the rise times are short and are measured in the range from 0.8 to $2 \mathrm{~ns}$, without a clear dependence on the fluorophore concentration (Table $\mathrm{S} 1 \dagger$ ). After the initial rise time, the resulting PL decay can be best fitted with a single long-lived $\tau_{\text {decay }}$ component for all films. ${ }^{36}$ The lack of additional recombination times, even at the higher concentrations, reflects the absence of dynamic processes attributed to aggregates within the films.

The fluorescence quantum yield and the mono-exponential decay lifetime of SilaFluo/PMMA films at different fluorophore content were plotted in Fig. 5 .

The maximum (and extremely high) value of QY was recorded for the lowest SilaFluo content, i.e. $85 \%$ for $0.1 \mathrm{wt} \%$ SilaFluo/PMMA film. By incrementing fluorophore content, a gradual and progressive drop of QY occurred with a maximum decrease seen within $0.2-0.8 \mathrm{wt} \%$, then stabilized to values around $65 \%$ for the highest SilaFluo content investigated and similarly to those reported in the literature. ${ }^{31}$ Values of quantum yield as those calculated for SilaFluo/PMMA films are considered promising for the realization of high efficiency LSC at wavelength emission higher than $600 \mathrm{~nm} .^{4,37}$

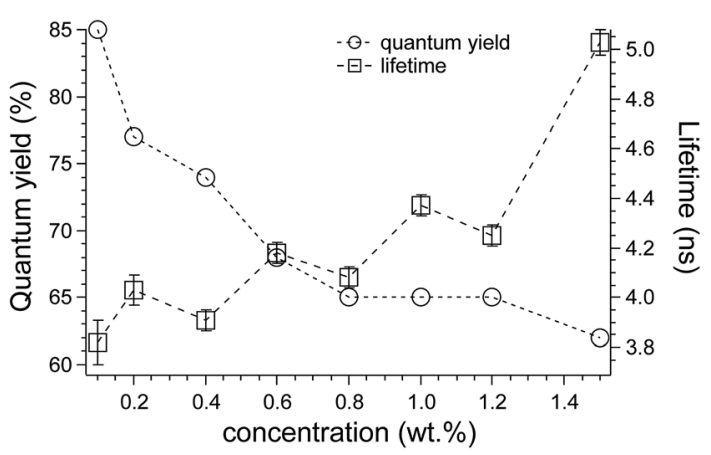

Fig. 5 Quantum yields and longer-lived lifetime of SilaFluo/PMMA films as a function of fluorophore concentration (wt\%).
An opposite trend was observed by looking at decay times. At the lowest fluorophore content, SilaFluo/PMMA films were characterized by lifetimes of $3.82 \pm 0.09$ ns. Notably, by increasing fluorophore content the recombination time of the long-lived population increases, reaching values of $5.03 \pm 0.05$ ns at the highest content of $1.5 \mathrm{wt} \%$. This increase at high fluorophore concentration can be ascribed to a more efficient population of the excited state, due to energy transfer from the donor moieties. However, inner filters or reabsorption effects, ${ }^{38}$ cannot be ruled out.

\section{Optical efficiency determination of SilaFluo/PMMA films}

Aiming at the determination of the optical efficiency and concentration factor of the selected SilaFluo/PMMA system, an optically pure $50 \times 50 \times 3 \mathrm{~mm}$ glass was coated with SilaFluo/ PMMA films with a thickness of $25 \pm 5 \mu \mathrm{m}$. Photocurrent measurements were accomplished with a home-built apparatus by using a Si-based PV cell sticked onto one edge of the fluorescent collector, as reported in the Experimental part. The optical efficiency $\eta_{\text {opt }}$ was determined as the ratio between the concentration factor $C$ and the geometrical factor $G$ of our device (i.e., $G=16.6$ ) (eqn (2)):

$$
\eta_{\mathrm{opt}}=\frac{C}{G}=\frac{I_{\mathrm{LSC}}}{I_{\mathrm{SC}} G}
$$

where $C$ is the ratio between the short circuit current measured in the case of the cell over the collector edge $\left(I_{\text {LSC }}\right)$ and short circuit current of the PV cell when perpendicular to the light source $\left(I_{\mathrm{SC}}\right)$ and $G$ is the ratio between the area exposed to the light source and the collecting area. The collected data were reported in Table 2 and compared to those gathered from luminescent collectors with the same geometry and thickness but containing Lumogen Red F350 (LR), as reference being considered the state-of-the-art in fluorophores for LSC

Table 2 Concentration factors (C) and optical efficiencies $\left(\eta_{\text {opt }}\right)$ calculated for SilaFluo/PMMA and LR/PMMA LSCs with different fluorophore contents (wt\%)

\begin{tabular}{lll}
\hline Entry & $C$ & $\eta_{\text {opt }}(\%)$ \\
\hline SilaFluo/PMMA (0.1 wt\%) & $1.02 \pm 0.08$ & 6.14 \\
SilaFluo/PMMA (0.2 wt\%) & $1.08 \pm 0.02$ & 6.50 \\
SilaFluo/PMMA (0.4 wt\%) & $1.13 \pm 0.06$ & 6.81 \\
SilaFluo/PMMA (0.6 wt\%) & $1.41 \pm 0.02$ & 8.49 \\
SilaFluo/PMMA (0.8 wt\%) & $1.47 \pm 0.06$ & 8.86 \\
SilaFluo/PMMA (1.0 wt\%) & $1.52 \pm 0.04$ & 9.16 \\
SilaFluo/PMMA (1.5 wt\%) & $1.60 \pm 0.05$ & 9.64 \\
SilaFluo/PMMA (1.6 wt\%) & $1.57 \pm 0.05$ & 9.46 \\
LR/PMMA (0.1 wt\%) & $0.83 \pm 0.01$ & 5.00 \\
LR/PMMA (0.2 wt\%) & $0.95 \pm 0.02$ & 5.72 \\
LR/PMMA (0.4 wt\%) & $1.04 \pm 0.05$ & 6.27 \\
LR/PMMA (0.6 wt\%) & $1.20 \pm 0.01$ & 7.22 \\
LR/PMMA (0.8 wt\%) & $1.09 \pm 0.02$ & 6.57 \\
LR/PMMA (1.0 wt\%) & $1.12 \pm 0.04$ & 6.75 \\
LR/PMMA (1.1 wt\%) & $1.22 \pm 0.06$ & 7.34 \\
LR/PMMA (1.2 wt\%) & $1.20 \pm 0.04$ & 7.23 \\
LR/PMMA (1.5 wt\%) & $1.22 \pm 0.04$ & 7.26
\end{tabular}


applications. ${ }^{4}$ Measurements of the LSC efficiencies are usually performed by considering solar insolation as referred to the recently introduced artificial AM1.5 spectrum of the National Renewable Energy Lab (NREL). ${ }^{1}$ While this approach is effective for evaluating the absolute luminescent collectors performance, it has created a lot of confusion in the literature data, ${ }^{\mathbf{4}}$ because many research groups make use of different and not always directly comparable conditions and experimental setups on their pursue to the best performing LSC system. Therefore, we preferred to use a home-built apparatus to compare the performances of the SilaFluo/PMMA system with those of the state-of-the-art.

The concentration factor $C$ and the optical efficiency $\eta_{\mathrm{opt}}$ were found to consistently increase as a function of SilaFluo concentration (Fig. 6), according to the progressive amount of dispersed fluorophore able to harvest UV-vis light irradiation. It is worth noting that this trend results only in part affected by dissipative phenomena, which are associated to the fluorescence quantum yield decreasing with concentration (Fig. 5). Actually, the highest value of $C(\sim 1.6$, which corresponds to $\sim 9.5 \%$ of $\eta_{\text {opt }}$ ) was reached at the $1.5 \mathrm{wt} \%$ of SilaFluo, after that a plateau was attained (Fig. 6a). These values are the highest ever registered in our laboratory with the same apparatus and LSC geometrical factor. ${ }^{\mathbf{9 , 2 0 , 2 2 , 3 2}}$ The outstanding performances of SilaFluo/PMMA system in terms of LSC characteristics were also confirmed when compared to those gathered from LSCs based on LR (Fig. 6a). Lower $C$ values were actually measured for the latter system for all the range of concentration, and the trend levelled off for samples at the highest LR content.

The greater performance of SilaFluo/PMMA LSCs in terms of light harvesting and concentration is clearly visible looking at the intensity of light escaping laterally from the fluorescent collector, when excited at $450 \mathrm{~nm}$ (Fig. 6b). These properties can be possibly attributed to a combination of effects: the first relies

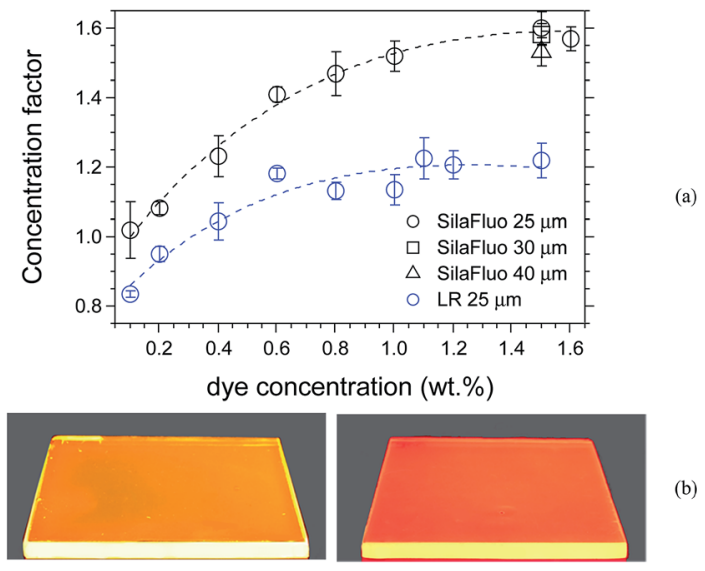

Fig. 6 (a) Concentration factor variation of $25 \mu \mathrm{m}$ thick SilaFluo/PMMA (black circles) and LR/PMMA (blue circles) LSCs as a function of fluorophore content. The black square and black triangle represent the 30 $\mu \mathrm{m}$ and $40 \mu \mathrm{m}$ thick SilaFluo/PMMA LSCs. The curves were fitted with eqn (3) with the parameters listed in Table 2; (b) pictures taken of 25 $\mu \mathrm{m}$ thick $1.5 \mathrm{wt} \%$ SilaFluo/PMMA (left) and $1.5 \mathrm{wt} \%$ LR/PMMA (right) LSCs under the excitation with a Dark Reader 46B transilluminator ( 450 nm). on the more intense absorption band of SilaFluo with respect to that of LR (see Fig. S10 $†$ ); the second resides on the possible higher SilaFluo compatibility with PMMA that limits dissipative phenomena of the overall emission occurring at high fluorophore content. ${ }^{39}$

Moreover, the effect of polymer thickness on the concentration efficiency of SilaFluo/PMMA LSCs was also investigated. As already reported earlier by our group, ${ }^{40}$ PMMA based films with $25 \mu \mathrm{m}$ of thickness were found to display the best trade-off for maximizing fluorophore concentration without adversely affecting the overall optical efficiencies. In agreement with these findings, the concentration factor of $1.5 \mathrm{wt} \%$ SilaFluo/ PMMA LSCs (i.e., the LSC with the highest optical performances) was found to be approximately the same for the $30 \mu \mathrm{m}$ thick film $(1.60 \pm 0.05$ against $1.55 \pm 0.04$ for $25 \mu \mathrm{m}$ and $30 \mu \mathrm{m}$ thick films, respectively). Conversely, it apparently decreased to $1.49 \pm 0.04$ for $40 \mu \mathrm{m}$ thick films, possibly due to more probable dye-dye interactions caused by the larger mean path length of the emitted radiation. Furthermore, while UV-vis absorption spectra of $1.5 \mathrm{wt} \%$ SilaFluo/PMMA films with thickness of 30 $\mu \mathrm{m}$ and $40 \mu \mathrm{m}$ experienced saturation (Fig. S11†), the fluorescence intensities resulted only slightly higher for the thicker film (Fig. S12 $\dagger$ ), although less than that measured for the $25 \mu \mathrm{m}$ thick at the same SilaFluo content (Fig. 3).

Finally, $C$ variations as a function of fluorophore content in PMMA were also fitted with eqn (3):

$$
C=\eta_{\mathrm{opt}} G \propto \varepsilon^{\prime} c \mathrm{e}^{-\mu_{\mathrm{opt}} c}+D
$$

where $\eta_{\text {opt }}$ is a term proportional to the current generated by the Si-based PV, $c$ is the concentration of the dye in wt\%, and $\varepsilon^{\prime}$ and $\mu_{\text {opt }}$ are two empirical parameters defined as:

$$
\begin{gathered}
\varepsilon^{\prime} \propto h \mathrm{e}^{-\bar{l}} \\
\mu_{\mathrm{opt}} \propto \mu^{\prime \prime}(\mathrm{QY}, p) \bar{l}
\end{gathered}
$$

where $h$ is the thickness of the thin film, $\bar{l}$ is the mean path length of the fluorescence in the LSC and $\mu^{\prime \prime}$ is a term depending on both QY and re-absorption probability $(p) . D$ is another empirical parameter that takes into account that even a transparent and clear material is capable of trapping light due to scattering phenomena from surface and bulk defects. Notably, $\varepsilon^{\prime}$ is a coefficient associated to the absorption features of the fluorophore/polymer system, whereas $\mu_{\text {opt }}$ integrates all the fluorescence quenching phenomena. Therefore, an ideal LSC should be endowed with high $\varepsilon^{\prime}$ and small $\mu_{\text {opt }}$ so that the maximum efficiency is shifted to higher fluorophore contents and the curve regularly rises due to the contribution of the linear part (eqn (3)). Eqn (3) is based on the work of Goetzberger $^{41}$ and it was also detailed described in our recent work. ${ }^{32}$ The fitting parameters of both curves are reported in Table 3.

The fitting parameters collected in Table 3 show different $\varepsilon^{\prime}$ and $\mu_{\text {opt }}$ values for SilaFluo/PMMA and LR/PMMA LSCs. Only $D$ values are equivalent for both system systems, being the contribution of non-fluorescent trapping almost the same for LSC with the same geometrical factor. Notably, the SilaFluo/ 
Table 3 Fitting parameters of the $C$ data gathered from SilaFluo/ PMMA and LR/PMMA LSCS

\begin{tabular}{llll}
\hline Entry & $\varepsilon^{\prime}$ & $\mu_{\text {opt }}$ & $D$ \\
\hline SilaFluo/PMMA & $1.22 \pm 0.10$ & $0.57 \pm 0.07$ & $0.80 \pm 0.05$ \\
LR/PMMA & $0.93 \pm 0.14$ & $0.95 \pm 0.15$ & $0.77 \pm 0.09$ \\
\hline
\end{tabular}

PMMA system seems to be characterized by a higher $\varepsilon^{\prime}$ thus suggesting that SilaFluo is capable to reach the highest photocurrent values at a lower concentration regime, possibly due to its more intense absorption in PMMA (Fig. S10 $\dagger$ ). Moreover, the smaller $\mu_{\text {opt }}$ value gathered from SilaFluo/PMMA with respect to LR/PMMA suggests that the former system is less affected by dissipative phenomena. This different performance can be addressed both to the better dispersability of SilaFluo fluorophores in PMMA also at high dye content, and also to its larger Stokes shifts (i.e., $116 \mathrm{~nm}$ for SilaFluo (Fig. 3) against $36 \mathrm{~nm}$ for LR (Fig. S13†)), that strongly contribute in preventing dye self-absorption and efficiency losses.

Photostability tests of the SilaFluo/PMMA films were eventually carried out by continuously irradiating a $0.25 \mathrm{~cm}^{2}$ area of the films at the wavelength of max absorbance (i.e. $480 \mathrm{~nm}$ ) with a $450 \mathrm{~W}$ Xe arc lamp under aerobic conditions. It is worth noting that the films retained the $96 \%$ of their fluorescence reaching a plateau after $60 \mathrm{~min}$ of continuous excitation (Fig. S14 $\dagger$ ), thus indicating excellent photostability, which makes SilaFluo a solid alternative to perylene-based fluorophores for LSC applications.

\section{Conclusions}

We have demonstrated that a red-emitting silafluorene with D-A features (SilaFluo) bestows PMMA transparent amorphous thin films excellent optical efficiencies that make them relevant for the preparation of high performance LSCs. Spectroscopic investigations revealed negligible aggregachromic behaviour both in solution and in the PMMA matrix, high stability upon light irradiation and limited auto-absorption phenomena as also confirmed by QY and lifetime experiments. Notably, the limited dissipative phenomena of the overall emission occurring at $1.5 \mathrm{wt} \%$ SilaFluo content for PMMA films with $25 \mu \mathrm{m}$ of thickness generates LSCs with optical efficiencies of maximum $9.5 \%$. i.e. at the top level respect to that gathered from the current state-of-art LSCs based on Lumogen Red.

\section{Acknowledgements}

The research leading to these results has received funding from the Università di Pisa under PRA 2017 (project No. 2017_28) and from the Ministry of Education, Culture, Sports, Science and Technology, Japan (Grant No. 15H03795 and 15H00740).

\section{Notes and references}

1 Photovoltaic Solar Energy Conversion. Lecture Notes in Physics, ed. G. H. Bauer, Springer, 2015, vol. 901.
2 N. Armaroli and V. Balzani, Chem.-Eur. J., 2016, 22, 32-57.

3 M. Debije, Nature, 2015, 519, 298-299.

4 M. G. Debije and P. P. C. Verbunt, Adv. Energy Mater., 2012, 2, 12-35.

5 J. L. Sawin, Renewables Global Status Report, Renewable Energy Policy Network for the 21th Century, Paris, 2014.

6 W. G. J. H. M. van Sark, Renewable Energy, 2013, 49, 207-210.

7 Research and Markets, BIPV Technologies and Markets 20152022, Dublin, Ireland, 2015.

8 Y. Zhou, D. Benetti, Z. Fan, H. Zhao, D. Ma, A. O. Govorov, A. Vomiero and F. Rosei, Adv. Energy Mater., 2016, 6, 1501913.

9 M. Carlotti, G. Ruggeri, F. Bellina and A. Pucci, J. Lumin., 2016, 171, 215-220.

10 R. Turrisi, A. Sanguineti, M. Sassi, B. Savoie, A. Takai, G. E. Patriarca, M. M. Salamone, R. Ruffo, G. Vaccaro, F. Meinardi, T. J. Marks, A. Facchetti and L. Beverina, J. Mater. Chem. A, 2015, 3, 8045-8054.

11 B. Valeur and M. N. Berberan-Santos, Molecular Fluorescence: Principles and Applications, Wiley-VCH, Weinheim, Germany, 2nd edn, 2013.

12 L. H. Slooff, E. E. Bende, A. R. Burgers, T. Budel, M. Pravettoni, R. P. Kenny, E. D. Dunlop and A. Büchtemann, Phys. Status Solidi RRL, 2008, 2, 257-259.

13 A. Sanguineti, M. Sassi, R. Turrisi, R. Ruffo, G. Vaccaro, F. Meinardi and L. Beverina, Chem. Commun., 2013, 49, 1618-1620.

14 B. McKenna and R. C. Evans, Adv. Mater., 2017, 29, DOI: 10.1002/adma.201606491.

15 A. Kaniyoor, B. McKenna, S. Comby and R. C. Evans, Adv. Opt. Mater., 2016, 4, 444-456.

16 R. Rondao, A. R. Frias, S. F. H. Correia, L. Fu, V. de Zea Bermudez, P. S. Andre, R. A. S. Ferreira and L. D. Carlos, ACS Appl. Mater. Interfaces, 2017, 9, 12540-12546.

17 F. Meinardi, S. Ehrenberg, L. Dhamo, F. Carulli, M. Mauri, F. Bruni, R. Simonutti, U. Kortshagen and S. Brovelli, Nat. Photonics, 2017, 11, 177-185.

18 Z. Krumer, W. G. J. H. M. van Sark, R. E. I. Schropp and C. d. M. Donega, Sol. Energy Mater. Sol. Cells, 2017, 167, 133-139.

19 N. J. L. K. Davis, R. W. MacQueen, S. T. E. Jones, C. OrofinoPena, D. Cortizo-Lacalle, R. G. D. Taylor, D. Credgington, P. J. Skabara and N. C. Greenham, J. Mater. Chem. C, 2017, 5, 1952-1962.

20 A. Pucci, F. De Nisi, A. Battisti, A. Panniello, E. Fanizza, R. Francischello, M. Striccoli, X. Gu, N. L. C. Leung and B. Z. Tang, Mater. Chem. Front., 2017, 1, 1406-1412.

21 A. Pucci, M. Pavone, P. Minei, A. B. Munoz-Garcia, E. Fanizza, P. Cimino and A. Rodriguez, $R S C$ Adv., 2016, 6, 17474-17482.

22 J. Lucarelli, M. Lessi, C. Manzini, P. Minei, F. Bellina and A. Pucci, Dyes Pigm., 2016, 135, 154-162.

23 A. Sharma, D. Pathak and T. Wagner, J. Optoelectron. Adv. Mater., 2014, 16, 1257-1268.

24 R. West, Pure Appl. Chem., 2008, 80, 563-569.

25 J. Chen and Y. Cao, Macromol. Rapid Commun., 2007, 28, 1714-1742. 
26 W. Ni, X. Wan, M. Li, Y. Wang and Y. Chen, Chem. Commun., 2015, 51, 4936-4950.

27 J. Y. Corey, Adv. Organomet. Chem., 2011, 59, 181-328.

28 M. Shimizu and T. Hiyama, Synlett, 2012, 23, 973-989.

29 W. W. H. Wong, J. F. Hooper and A. B. Holmes, Aust. J. Chem., 2009, 62, 393-401.

30 W. W. H. Wong and A. B. Holmes, in Advances in Polymer Science, ed. U. Scherf and D. Neher, Springer, Berlin, Heidelberg, 2008, pp. 85-98.

31 M. Shimizu, K. Mochida, M. Katoh and T. Hiyama, J. Phys. Chem. C, 2010, 114, 10004-10014.

32 M. Carlotti, E. Fanizza, A. Panniello and A. Pucci, Sol. Energy, 2015, 119, 452-460.

33 H. Zhu, R. Derksen, C. Krause, R. Fox, R. Brazee and H. Ozkan, J. ASTM Int., 2005, 2, 1-7.

34 F. Donati, A. Pucci, C. Cappelli, B. Mennucci and G. Ruggeri, J. Phys. Chem. B, 2008, 112, 3668-3679.
35 J. R. Lakowicz, Principles of Fluorescence Spectroscopy, Springer, Berlin/Heidelberg, Germany, 3rd edn, 2006.

36 M. A. Wolak, J. S. Melinger, P. A. Lane, L. C. Palilis, C. A. Landis, J. E. Anthony and Z. H. Kafafi, J. Phys. Chem. $B, 2006,110,10606-10611$.

37 V. M. Granchak, T. V. Sakhno and S. Y. Kuchmy, Theor. Exp. Chem., 2014, 50, 1-20.

38 S. Dhami, A. J. D. Mello, G. Rumbles, S. M. Bishop, D. Phillips and A. Beeby, Photochem. Photobiol., 1995, 61, 341-346.

39 C. Tummeltshammer, A. Taylor, A. J. Kenyon and I. Papakonstantinou, Sol. Energy Mater. Sol. Cells, 2016, 144, 40-47.

40 M. Carlotti, A. Panniello, E. Fanizza and A. Pucci, Sol. Energy, 2015, 119, 452-460.

41 A. Goetzberger and W. Greube, Appl. Phys., 1977, 14, 123139. 